\title{
Familiar face + novel face $=$ familiar face? Representational bias in the perception of morphed faces in chimpanzee
}

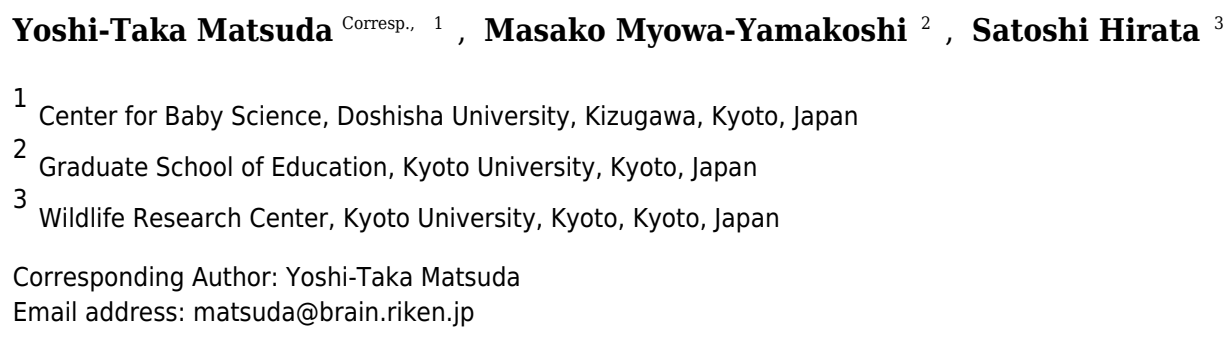

Highly social animals possess a well-developed ability to distinguish the faces of familiar from novel conspecifics to induce distinct behaviors for maintaining society. However, the behaviors of animals when they encounter ambiguous faces of familiar yet novel conspecifics, e.g., strangers with faces resembling known individuals, have not been well characterised. Using a morphing technique and preferential-looking paradigm, we address this question via the chimpanzee's facial-recognition abilities. We presented eight subjects with three types of stimuli: (1) familiar faces, (2) novel faces and (3) intermediate morphed faces that were $50 \%$ familiar and $50 \%$ novel faces of conspecifics. We found that chimpanzees spent more time looking at novel faces and scanned novel faces more extensively than familiar or intermediate faces. Interestingly, chimpanzees looked at intermediate faces in a manner similar to familiar faces with regards to the fixation duration, fixation count, and saccade length for facial scanning, even though the participant was encountering the intermediate faces for the first time. We excluded the possibility that subjects merely detected and avoided traces of morphing in the intermediate faces. These findings suggest a bias for a feeling-of-familiarity that chimpanzees perceive familiarity with an intermediate face by detecting traces of a known individual, as $50 \%$ alternation is sufficient to perceive familiarity. 
1 Familiar face + novel face $=$ familiar face? Representational bias in the

2 perception of morphed faces in chimpanzees

3

4 Yoshi-Taka Matsuda ${ }^{1 *}$, Masako Myowa-Yamakoshi² and Satoshi Hirata ${ }^{3 *}$

5

$6 \quad{ }^{1}$ Center for Baby Science, Doshisha University, Kizugawa, Japan

$7 \quad 2$ Graduate School of Education, Kyoto University, Kyoto, Japan

$8{ }^{3}$ Kumamoto Sanctuary, Wildlife Research Center, Kyoto University, Uki, Japan

9

10 *Authors for correspondence:

11 Yoshi-Taka Matsuda: Center for Baby Science, Doshisha University, 4-1-1 Kizugawadai,

12 Kizugawa, Kyoto 619-0225 Japan. Tel: +81-774-65-6066, E-mail: matsuda@brain.riken.jp

13 Satoshi Hirata: Kumamoto Sanctuary, Wildlife Research Center, Kyoto University, 990 Otao,

14 Misumi, Uki, Kumamoto 869-3201 Japan. Tel: +81-964-34-1130, E-mail:

$15 \quad$ hirata.satoshi.8z@kyoto-u.ac.jp 


\section{Summary}

17 Highly social animals possess a well-developed ability to distinguish the faces of familiar from

18 novel conspecifics to induce distinct behaviours for maintaining society. However, the

19 behaviours of animals when they encounter ambiguous faces of familiar yet novel conspecifics,

20 e.g., strangers with faces resembling known individuals, have not been well characterised. Using

21 a morphing technique and preferential looking paradigm, we address this question via the

22 chimpanzee's facial recognition abilities. We presented eight subjects with three types of stimuli:

23 (1) familiar faces, (2) novel faces and (3) intermediate morphed faces that were $50 \%$ familiar and

$2450 \%$ novel faces of conspecifics. We found that chimpanzees spent more time looking at novel

25 faces and scanned novel faces more extensively than familiar or intermediate faces. Interestingly,

26 chimpanzees looked at intermediate faces in a manner similar to familiar faces with regards to

27 the fixation duration, fixation count, and saccade length for facial scanning even though the

28 participant was encountering the intermediate faces for the first time. We excluded the possibility

29 that subjects merely detected and avoided traces of morphing in the intermediate faces. These

30 findings suggest a bias for a feeling of familiarity and that chimpanzees perceive familiarity with

31 an intermediate face by detecting traces of a known individual; $50 \%$ alternation is sufficient to 
32 perceive familiarity.

33

PeerJ reviewing PDF | (2015:07:6096:2:0:NEW 7 Jul 2016) 


\section{Introduction}

35 Distinction between in- and out-group members based on faces has greatly influenced the

36 evolution of society, leading to individualised relationships and cooperative networks (Hamilton,

37 1964). Notably, many social animals can distinguish between familiar and unfamiliar faces of

38 conspecifics, such as humans (Bentin, Allison, Puce, Perez, \& McCarthy, 1996; Rossion, Schiltz,

39 \& Crommelinck, 2003), chimpanzees (Boysen \& Berntson, 1989; Fukushima et al., 2013; Parr,

40 Winslow, Hopkins, \& de Waal, 2000), orangutans (Hanazuka, Shimahara, Tokuda, \&

41 Midorikawa, 2013; Vonk \& Hamilton, 2014), gorilla (Vonk \& Hamilton, 2014), rhesus monkeys

42 (Gothard, Erickson, \& Amaral, 2004; Murai, Tanaka, Tomonaga, \& Sakagami, 2011), capuchin

43 monkeys (Pokorny \& de Waal, 2009), dogs (Racca et al., 2010), sheep (Kendrick, da Costa,

44 Leigh, Hinton, \& Peirce, 2001), cattle (Coulon, Deputte, Heyman, \& Baudoin, 2009), pigeons

45 (Wilkinson, Specht, \& Huber, 2010) and even invertebrates (Chittka \& Dyer, 2012). individuals. Theoretically, three behavioural outcomes are possible: animals could behave 
50 as the novel faces or 3) in a manner different from both the novel and familiar faces. A previous

51 study showed that human adults perceive familiarity towards composite faces of novel and

52 familiar (self, sibling and friend) individuals (Platek \& Kemp, 2009), which supports the first

53 possible outcome among the above-mentioned possibilities. This study suggests that facial

54 resemblance acts as a cue for genetic relatedness (Daly, 1988) and increases ratings of

55 trustworthiness (DeBruine, 2002). A bias towards novelty in responses to composite faces (the

56 second possible outcome) is supported by the attention model of categorisation (Kruschke, 2003)

57 or hypodescent (Halberstadt, Sherman, \& Sherman, 2011): attention is strongly tuned towards

58 the features that best distinguish between in- and out-group members while ignoring shared

59 features, i.e., the assignment of ambiguous faces to out-group members. We previously showed

60 that human infants have a lower preference for composite faces, which include aspects of

61 familiar and novel individuals, than original faces (Matsuda, Okamoto, Ida, Okanoya, \& Myowa-

62 Yamakoshi, 2012) (the third possibility discussed above). This study suggests that a negative

63 feeling (or avoidant response) occurs because the composite faces elicit the concept of

64 'familiarity' but fail to satisfy it (e.g., (Mori, 1970);(Steckenfinger \& Ghazanfar, 2009). It

65 remains unknown whether other primates exhibit familiarity-biased responses, novelty-biased 
66 responses or negative responses when they encounter ambiguous faces. Answering this question

67 may aid our understanding of the evolutionary and developmental profiles of facial recognition

68 in detecting the category boundary between in- and out-group members. morphing technique and a preferential looking paradigm. As is the case with human species,

71 chimpanzees are known to possess a well-developed ability to readily individualise faces (Dahl,

72 Rasch, Tomonaga, \& Adachi, 2013; Hirata, Fuwa, Sugama, Kusunoki, \& Fujita, 2010; Kano \&

73 Tomonaga, 2009; Tomonaga, 2007) and distinguish between familiar versus novel conspecifics

74 (Fukushima et al., 2013) even when shown only static two-dimensional images that are absent

75 any other cues (e.g., olfaction). They also have the ability to identify individuals in terms of

76 kinship detection via phenotypic matching (Parr \& de Waal, 1999; Parr, Heintz, Lonsdorf, \&

77 Wroblewski, 2010). Furthermore, chimpanzees show categorical perception in face recognition;

78 that is, they detect a category boundary on a spectrum-ranging morphed continuum that

79 comprises two different faces, and their sensitivity for categorisation is affected by exposure to

80 conspecific/non-conspecific faces during development (Martin-Malivel \& Okada, 2007). The

81 morphing technique has the advantage of creating composite portraits between two faces with 
82 physical accuracy in blend ratios, which enables us to manipulate and quantify the degrees of

83 similarity between different faces (DeBruine, Jones, Little, \& Perrett, 2008; Matsuda et al., 2012;

84 Myowa-Yamakoshi, Yamaguchi, Tomonaga, Tanaka, \& Matsuzawa, 2005; Parr, Taubert, Little,

$85 \&$ Hancock, 2012).

86 In contrast to the discrimination paradigm, the preferential looking paradigm with free

87 viewing has the advantage of investigating intrinsic gazing behaviours such as preference,

88 attention or processing costs by minimising training effects and testing beyond the limits of a

89 match-to-sample task, as we reported previously in adult and infant chimpanzees (Hirata et al.,

90 2010). The aim of our study was to quantify the degree of similarity between faces that

91 chimpanzees perceive as belonging to familiar conspecifics. We investigated the gazing

92 behaviour of chimpanzees when presented with composite faces representing morphs between

93 familiar and novel conspecifics. First, we presented 'intermediate faces' (50\% familiar and 50\%

94 novel faces) to chimpanzees and examined whether they preferred these intermediate conspecific

95 faces to the original faces. We identified the faces preferred by the chimpanzee when presented

96 with three pairs of faces: familiar versus novel faces, familiar versus intermediate faces and

97 novel versus intermediate faces. 
98 Given that novel faces (i.e., potential threats) should elicit more of a response from the

99 chimpanzees than familiar faces (Pascalis \& Bachevalier, 1998), we measured the time (fixation

100 duration) spent looking at a face, the frequency (fixation count) with which the chimpanzee

101 looked at the face, and the saccade length for how extensively they scan the face; these indices

102 were used to measure the attentional and processing demand/efficacy (i.e., informativeness)

103 (Antes, 1974; Henderson, 2003; Henderson \& Smith, 2009; Loftus \& Mackworth, 1978;

104 McCamy, Otero-Millan, Di Stasi, Macknik, \& Martinez-Conde, 2014; Unema, Pannasch, Joos,

$105 \&$ Velichkovsky, 2005) during facial recognition and to differentiate gazing behaviours

106 associated with viewing familiar and novel faces. 


\section{Methods}

110 Laboratory Animals of Hayashibara Biochemical Laboratories, Inc. and the Weatherall Report,

111 The use of non-human primates in research. The research protocol was approved by the Animal

112 Welfare and Animal Care Committee of the Hayashibara Great Ape Research Institute (GARI-

113 051101).

\section{Participants}

117 participated in this study. The names, ages, and sexes of the chimpanzees were shown in the

118 table 1. The subjects were members of a captive group at the Great Ape Research Institute

119 (Hayashibara Biomedical Laboratories, Inc.). Relative dominances and kinship structures were

120 shown in the tables S1 and S2. Loi, Zamba, Tsubaki, and Mizuki came to the institute from other

121 locations when they were 3,3,3, and 2 years old, respectively, and since arriving have spent

122 their time together in the same group. Misaki was added to the group when she was 3 years old.

123 Since then, she has spent her time with the above-mentioned individuals in the same enclosure, 
124 with few exceptions (described below). Natsuki was born to Tsubaki, and Iroha was born to

125 Mizuki. These offspring grew up in the same group with their mothers and other group members.

126 Hatsuka is a daughter of Misaki but was neglected between the ages of 40 days and 2.5 years.

127 During this period, human caretakers nursed Hatsuka, but she spent 10 minutes to 3 hours of

128 nearly every day with all of the other chimpanzees in the same space. Misaki spent all of her

129 time with the other chimpanzees (except her daughter) during this period. After the age of 2.5

130 years, Hatsuka spent all of her time with Misaki (her mother), and the two of them were

131 separated from the other members in an adjacent enclosure. However, they maintained visual

132 contact with the other members in the neighbouring enclosure through wire mesh or transparent

133 panels. In short, the adult participants (Loi, Zamba, Tsubaki, Mizuki, and Misaki) had known

134 each other since they were 2-3 years old, for approximately 10 years or more, and the adolescent

135 (Natsuki) and infant (Hatsuka and Iroha) individuals were born into this group and had known

136 each other and the other group members since they were born.

Three individuals, whose faces were presented to the subjects as familiar-face stimuli,

138 also participated in the study (Loi, Misaki and Zamba). Although these three individuals saw

139 their own faces on a screen in the experiment, our previous study using the 
140 electroencephalography (EEG) recording showed that no significant difference was observed

141 between self- and familiar-face recognition (Fukushima et al., 2013). This finding may be caused

142 by past experiences with informal tests of mirror self-recognition and recognition of self-images

143 on TV monitors (Hirata, Fuwa, \& Myowa-Yamakoshi, in preparation). Thus, we presented self-

144 face stimuli as 'familiar' faces to the three individuals. This is consistent with a previous study

145 that showed animals failed to treat self images any differently than other familiar faces, which

146 suggests that the subjects found self images to be familiar given the glass and other reflective

147 surfaces present in their outdoor habitats (Vonk \& Hamilton, 2014).

149 stimuli were tabulated (Table S1-2).

150

151

\section{Experimental apparatus}

The subjects sat in an experimental room $(3 \mathrm{~m} \times 2 \mathrm{~m} \times 2.5 \mathrm{~m}, \mathrm{~L} \times \mathrm{W} \times \mathrm{H})$ and viewed

154 the images on a 17 -inch LCD screen $(1024 \times 768$ pixels $)$ at a distance of $60 \mathrm{~cm}$. The eye movements of the chimpanzees were recorded using a table-mounted eye tracker (Tobii T60, 
156 Stockholm, Sweden) (Hirata et al., 2010).

157

158 Stimuli description Prior to the experiments, coloured and frontal-orientation photographs of familiar (in-

160 group) chimpanzees and novel chimpanzees (from an out-group housed at the Kumamoto

161 Sanctuary, Wildlife Research Centre, Kyoto University) were taken. Each photograph was

162 rotated in-plane to horizontally align the interpupil distance and was then resized to $512 \times 680$

163 pixels (at a distance of $60 \mathrm{~cm}$ with approximately $13.3 \times 17.4$ degrees of visual angle). Using

164 computer morphing software (Sqirlz Morph 2.1: Xiberpix, Solihull, UK, www.xiberpix.com),

165 approximately 350 points were positioned on each chimpanzee face (approximately 100 points

166 for facial contour and 250 for facial parts) to delineate specific facial landmarks (e.g., distinctive

167 wrinkles under the eyes, across the muzzle, and in the ear region) (DeBruine et al., 2008; Parr et

168 al., 2012). To create intermediate faces, a familiar face and a novel face were morphed together

169 using the software (Sqirlz Morph 2.1) to produce a new face incorporating 50\% of the familiar

170 face and $50 \%$ of the novel face by calculating the mean shape and colour of the constituents

171 (Figure 1) (Matsuda et al., 2012). A previous study showed that chimpanzees could detect facial 
172 similarities between mothers and sons but not between mothers and daughters (Parr \& de Waal,

173 1999), which suggests that chimpanzees are better at detecting facial similarities among males;

174 consequently, we morphed two photographs of male chimpanzees to create an intermediate face.

175 Intermediate faces were created from photographs of different chimpanzees than those used as

176 the familiar face and novel face stimuli; this step was taken to prevent an adaptation effect that

177 could occur when the chimpanzees were repeatedly presented with images of the same faces

178 (even though the morphs bore only partial resemblance to the original faces).

179 We used movie clips of dynamic facial expressions as visual stimuli because primates

180 and humans are more responsive to moving faces than to static faces (Shepherd, Steckenfinger,

181 Hasson, \& Ghazanfar, 2010). We created moving stimuli of mouth-opening chimpanzee faces

182 that were familiar, novel or intermediate; these stimuli are known as dynamic facial expressions

183 or dynamic faces (Kilts, Egan, Gideon, Ely, \& Hoffman, 2003; LaBar, Crupain, Voyvodic, \&

184 McCarthy, 2003). Moving stimuli were created in the following manner: first, two coloured

185 photographs (mouth-closing and mouth-opening faces) of each individual chimpanzee were

186 taken prior to the main experiment. Next, for each participant, ten intermediate images with

187 expressions situated between the close-mouthed and open-mouthed expressions were created in 
$1889 \%$ steps using the computer-morphing techniques. Then, to create a moving clip, the 12 images

189 (one mouth-closing image, 10 intermediate images and the final mouth-opening image) were

190 presented in succession. Each image was presented for $40 \mathrm{~ms}$, and the final image was presented

191 for an additional $760 \mathrm{~ms}$; thus, each animation clip lasted 1,200 ms. Each clip was shown five

192 times (i.e., totalling a six-second duration) in both the main and control experiments (see video

193 clips of examples in the electronic supplementary materials; familiar-face.avi, intermediate-

194 face.avi and novel-face.avi). A human expert viewed the images to confirm that the presentation

195 speed sufficiently reflected the natural changes in the dynamic facial expressions of

196 chimpanzees.

197

198

199 Experimental procedure and data analysis

201 Two-point automated calibration was conducted by presenting a movie clip on each reference

202 point. A relatively small number of reference points was adopted for the chimpanzees because

203 they tended to view these reference points only briefly and no training procedure was adopted for 
204 them. However, we checked the accuracy after the initial calibration and repeated the calibration

205 if necessary. Our validation session confirmed the comparable accuracy between chimpanzees

206 and humans (see (Hirata et al., 2010; Kano, Hirata, Call, \& Tomonaga, 2011); the calibration

207 errors were $0.2-1$ degree in both chimpanzees and humans). Then, the chimpanzees were

208 presented with the following four pairs of stimuli: (1) familiar versus novel faces, (2) familiar

209 versus intermediate faces, (3) novel versus intermediate faces and (4) novel versus morphed

210 faces of two novel chimpanzees. In each of the four trials, a pair of faces was presented side-by-

211 side on an eye tracker screen for six seconds. Each novel chimpanzee face was presented only

212 once to prevent an adaptation effect. Each trial was preceded by a stimulus intended to attract the

213 participant's visual attention to the centre of the screen. The order of the four test trials and the

214 side on which a given face appeared was random and counterbalanced across participants.

215 In the data analysis, entire faces of presented stimuli were chosen as areas of interest

216 rather than specific facial parts because we used movie stimuli of dynamic facial expressions as

217 mentioned above. We measured the total looking durations, counts and saccade lengths for each

218 face. The saccade length was measured as a sum of the looking path length during the face

219 scanning for each face. We averaged the same types of facial stimuli for each participant to 
220 minimise the variations in looking behaviours that occasionally appear during free viewing. The

221 data were normalised to calculate proportions between three types of facial stimuli (familiar,

222 intermediate and novel faces for Figure 1) and between two types of facial stimuli (novel and

223 morphed faces of two novel faces for Figure 2) (Matsuda et al., 2012). We omitted data if the

224 chimpanzee (participant) looked at only one side of a pair of stimulus faces (strong side bias; see

225 chimpanzee \#4 eye tracking data in the supplementary information for Figure 2). 


\section{Results}

228 Figure 1a depicts an example of the three different types of stimuli: a familiar face, an

229 intermediate face and a novel face. The fixation duration, fixation count and saccade length for

230 each image are shown in Figure 1b, 1c and 1d, respectively. The non-parametric Friedman test

231 for all participants $(\mathrm{N}=8)$ revealed significant overall effects for the fixation duration $\left(\chi^{2}(2)=\right.$

$2326.25, N=8, p=0.04$; Figure $1 \mathrm{~b})$, for the fixation count $\left(\chi^{2}(2)=7.47, N=8, p=0.02\right.$; Figure 1c)

233 and for the saccade length $\left(\chi^{2}(2)=9.25, N=8, p=0.01\right.$; Figure 1d). The non-parametric

234 Wilcoxon signed-rank test (two-tailed) showed that the fixation count and saccade length were

235 significantly different between the familiar and novel faces $(Z=-2.24, N=8, p=0.02$ for the

236 fixation count and $Z=-2.52, N=8, p=0.01$ for the saccade length), whereas the fixation

237 duration was similar $(Z=-1.68, N=8, p=0.11)$; all indices were significantly different for the

238 intermediate and novel faces $(Z=-2.52, N=8, p<0.01$ for the fixation duration; $Z=-2.24, N=$

$2398, p<0.03$ for the fixation count; $Z=-2.38, N=8, p<0.02$ for the saccade length). No

240 significant differences in these indices were found for the familiar and intermediate faces $(Z=$

$2410.70, N=8, p=0.55$ for the fixation duration; $Z=-0.35, N=8, p=0.78$ for the fixation count; $Z$

$242=0.14, N=8, p=0.95$ for the saccade length). These results suggest that novel faces elicit a 
243 greater response than familiar or intermediate faces.

244 Although intermediate faces were less preferred than novel faces, it is possible that the

245 chimpanzees detected traces of morphing (e.g., a blur in wrinkles) and subsequently avoided the

246 unnatural-looking intermediate faces. In this case, the chimpanzees should show a lower

247 preference for the morphed faces per se, irrespective of constituent individuals or whether a

248 familiar or novel face was shown. To exclude this possibility, we presented the subjects with an

249 image of a novel chimpanzee and an image of a morphed face between two different novel

250 chimpanzees. The duration spent looking at the two faces and the frequency with which the faces

251 were observed did not differ significantly, nor did the saccade length for facial scanning

252 (Wilcoxon signed-rank test (two-tailed), $Z=1.52, N=7, p=0.16$ for the fixation duration,

253 Figure 2a; $Z=1.18, N=7, p=0.30$ for the fixation count, Figure $2 \mathrm{~b} ; Z=0.68, N=7, p=0.58$

254 for the saccade length, Figure 2c). This result indicates that the participants neither detected nor

255 avoided the morphed faces; instead, they showed a lower preference for the intermediate faces

256 than the novel faces because of constituent familiar faces.

We found systematic differences for participants in the perception of facial parts and

258 body (the eyes, mouth and body of stimuli were defined as areas of interest); the non-parametric 
259 Friedman test for all participants revealed significant overall effects for the fixation duration

$260\left(\chi^{2}(2)=14.25, N=8, p=0.0008\right)$. The non-parametric Wilcoxon signed-rank test (two-tailed)

261 showed that the fixation duration was significantly different between the eyes and mouth $(Z=-$

$2622.52, N=8, p=0.008)$ and between the mouth and body $(Z=2.52, N=8, p=0.008)$ but not

263 between the eyes and body $(Z=1.40, N=8, p=0.20)$. Larger attention to the mouth part of

264 facial stimuli may result from the presentation of movie stimuli of mouth-opening faces. 


\section{Discussion}

270 In contrast with our previous human study (Matsuda et al., 2012), chimpanzees did not exhibit

271 negative/ avoidant responses to intermediate faces (i.e., 50-50\% composite faces between

272 familiar and novel conspecifics). This difference occurred largely because the chimpanzees paid

273 less attention to the familiar faces and preferred the novel faces. This novelty preference for

274 familiar faces appeared as early as approximately $200 \mathrm{~ms}$ after stimulus presentation (Figure S1

275 in the supplementary information), which is consistent with our previous EEG study that showed

276 that different neural responses to familiar and novel faces have a latency of approximately 200

277 ms (Fukushima et al., 2013).

279 intermediate faces to a similar extent. One possible reason for this outcome was that morphing

280 made a face more similar to the prototype and, therefore, made the face more attractive (Langlois

281 \& Roggman, 1990); however, this was not the case. Rather, the results indicate that the

282 chimpanzees detected the resemblance between the intermediate face and the familiar face and

283 thus responded to these faces in a similar manner. In other words, the category boundary

284 between familiar (i.e., in-group) and novel (i.e., out-group) faces is more closely situated towards 
285 novel faces on a spectrum ranging from the familiar to the novel. This claim is consistent with a

286 previous study of morphed-face recognition in humans that showed a bias in favour of a feeling

287 of familiarity when one encounters intermediate faces (50-50\%) of self-, sibling- and friend-

288 stranger morphing (Platek \& Kemp, 2009).

The ability to detect facial resemblance may play a key role in kinship recognition.

290 Previous studies illustrated that chimpanzees are capable of visually recognising kin from

291 pictures of novel individuals (Parr \& de Waal, 1999; Parr et al., 2010). These previous studies,

292 however, used a matching-to-sample paradigm, and the chimpanzees went through basic training

293 of the match-to-sample task for familiar related individuals; thus, they may have simply learned

294 to detect individuals with facial resemblances (Parr \& de Waal, 1999; Parr et al., 2010). Using a

295 free-viewing paradigm, we demonstrated that chimpanzees voluntarily detect individuals with

296 facial resemblances.

298 faces (i.e., morphs between familiar (mother) and novel (stranger) faces) than for original faces

299 (Matsuda et al., 2012), which is different from the results of the chimpanzees in the present study.

300 This variation may originate from differences in the species, the age of the chimpanzees and/or 
301 the target faces. Thus, it is difficult to directly compare our results from human infants and adult

302 chimpanzees without considering species similarities and differences in their cognitive

303 development and adaptive significance. For example, a previous study showed that infant

304 chimpanzees preferentially looked at their mother's face, the most familiar face to the infants,

305 over a computer-created, average face (i.e., novel face) when they were between 4 and 8 weeks

306 of age; however, such a preference for the mother's face suddenly disappeared after 8 weeks of

307 age (Myowa-Yamakoshi et al., 2005). The developmental change in the mother/stranger

308 preference has also been observed in human infants (Gredeback, Fikke, \& Melinder, 2010). This

309 may be because both chimpanzee and human infants need to recognise their caregiver during the

310 initial phase of their development (it is vital for their survival); however, infants become

311 socialised at a certain stage by engaging in interactions with individuals other than their mothers

312 (Matsuzawa, Tomonaga, \& Tanaka, 2006). In short, it is natural to assume that preferences for

313 certain faces are affected by various factors, including development and the environment (Dahl

314 et al., 2013; Martin-Malivel \& Okada, 2007). 
317 a representation bias in favour of a feeling of familiarity when one encounters intermediate faces

318 between in- and out-group conspecifics.

319

320

321 


\section{References}

324 Antes, J. R. (1974). The time course of picture viewing. J Exp Psychol, 103(1), 62-70.

325 Bentin, S., Allison, T., Puce, A., Perez, E., \& McCarthy, G. (1996). Electrophysiological Studies

326 of Face Perception in Humans. J Cogn Neurosci, 8(6), 551-565.

327 Boysen, S. T., \& Berntson, G. G. (1989). Conspecific recognition in the chimpanzee (Pan troglodytes): cardiac responses to significant others. J Comp Psychol, 103(3), 215-220.

Chittka, L., \& Dyer, A. (2012). Cognition: Your face looks familiar. Nature, 481(7380), 154155. PLoS One, 4(2), e4441. perception. Sci Rep, 3, 1044. 
340 DeBruine, L. M., Jones, B. C., Little, A. C., \& Perrett, D. I. (2008). Social perception of facial resemblance in humans. Arch Sex Behav, 37(1), 64-77.

342 Fukushima, H., Hirata, S., Matsuda, G., Ueno, A., Fuwa, K., \& al., e. (2013). Neural representation of face familiarity in an awake chimpanzee. PeerJ, 1, e223.

344 Gothard, K. M., Erickson, C. A., \& Amaral, D. G. (2004). How do rhesus monkeys ( Macaca mulatta) scan faces in a visual paired comparison task? Anim Cogn, 7(1), 25-36.

346 Gredeback, G., Fikke, L., \& Melinder, A. (2010). The development of joint visual attention: a longitudinal study of gaze following during interactions with mothers and strangers. Dev Sci, 13(6), 839-848.

Halberstadt, J., Sherman, S. J., \& Sherman, J. W. (2011). Why Barack Obama Is Black A Cognitive Account of Hypodescent. Psychol Sci, 22(1), 29-33.

351 Hamilton, W. D. (1964). The genetical evolution of social behaviour. II. J Theor Biol, 7(1), 1752.

353 Hanazuka, Y., Shimahara, N., Tokuda, Y., \& Midorikawa, A. (2013). Orangutans (Pongo pygmaeus) remember old acquaintances. PLoS One, 8(12), e82073. 
355 Henderson, J. M. (2003). Human gaze control during real-world scene perception. Trends Cogn

356

357

358

359

360

361

362

363

364

365

366

367

368

369

370
Sci, 7(11), 498-504.

Henderson, J. M., \& Smith, T. J. (2009). How are eye fixation durations controlled during scene viewing? Further evidence from a scene onset delay paradigm. Vis Cogn, 17(6-7), 10551082.

Hirata, S., Fuwa, K., Sugama, K., Kusunoki, K., \& Fujita, S. (2010). Facial perception of conspecifics: chimpanzees (Pan troglodytes) preferentially attend to proper orientation and open eyes. Anim Cogn, 13(5), 679-688.

Kano, F., Hirata, S., Call, J., \& Tomonaga, M. (2011). The visual strategy specific to humans among hominids: A study using the gap-overlap paradigm. Vision research, 51(23), 2348-2355.

Kano, F., \& Tomonaga, M. (2009). How chimpanzees look at pictures: a comparative eyetracking study. Proc Biol Sci, 276(1664), 1949-1955.

Kendrick, K. M., da Costa, A. P., Leigh, A. E., Hinton, M. R., \& Peirce, J. W. (2001). Sheep don't forget a face. Nature, 414(6860), 165-166.

Kilts, C. D., Egan, G., Gideon, D. A., Ely, T. D., \& Hoffman, J. M. (2003). Dissociable neural 
pathways are involved in the recognition of emotion in static and dynamic facial

372 expressions. Neuroimage, 18(1), 156-168.

373 Kruschke, J. K. (2003). Attention in learning. Curr Dir Psychol Sci, 12(5), 171-175.

374 LaBar, K. S., Crupain, M. J., Voyvodic, J. T., \& McCarthy, G. (2003). Dynamic perception of facial affect and identity in the human brain. Cereb Cortex, 13(10), 1023-1033. $115-121$.

Loftus, G. R., \& Mackworth, N. H. (1978). Cognitive determinants of fixation location during picture viewing. J Exp Psychol Hum Percept Perform, 4(4), 565-572.

Martin-Malivel, J., \& Okada, K. (2007). Human and chimpanzee face recognition in chimpanzees (Pan troglodytes): role of exposure and impact on categorical perception. Behav Neurosci, 121(6), 1145-1155.

Matsuda, Y. T., Okamoto, Y., Ida, M., Okanoya, K., \& Myowa-Yamakoshi, M. (2012). Infants prefer the faces of strangers or mothers to morphed faces: an uncanny valley between social novelty and familiarity. Biol Lett, 8(5), 725-728. 
Springer.

388

389

390

391

392

393

394

395

396

397
McCamy, M. B., Otero-Millan, J., Di Stasi, L. L., Macknik, S. L., \& Martinez-Conde, S. (2014). Highly informative natural scene regions increase microsaccade production during visual scanning. J Neurosci, 34(8), 2956-2966.

Mori, M. (1970). The uncanny valley. Energy, 7(33-35).

Murai, C., Tanaka, M., Tomonaga, M., \& Sakagami, M. (2011). Long-term visual recognition of familiar persons, peers, and places by young monkeys (Macaca fuscata). Dev Psychobiol, 53(7), $732-737$.

Myowa-Yamakoshi, M., Yamaguchi, M. K., Tomonaga, M., Tanaka, M., \& Matsuzawa, T. (2005). Development of face recognition in infant chimpanzees (Pan troglodytes). Cogn Dev, 20, 49-63.

Parr, L. A., \& de Waal, F. B. (1999). Visual kin recognition in chimpanzees. Nature, 399(6737), 647-648.

Parr, L. A., Heintz, M., Lonsdorf, E., \& Wroblewski, E. (2010). Visual Kin Recognition in Nonhuman Primates: (Pan troglodytes and Macaca mulatta): Inbreeding Avoidance or Male Distinctiveness? J Comp Psychol, 124(4), 343-350. 
403 Parr, L. A., Taubert, J., Little, A. C., \& Hancock, P. J. (2012). The organization of conspecific

404 face space in nonhuman primates. QJ Exp Psychol (Hove), 65(12), 2411-2434.

405 Parr, L. A., Winslow, J. T., Hopkins, W. D., \& de Waal, F. B. (2000). Recognizing facial cues:

406 individual discrimination by chimpanzees (Pan troglodytes) and rhesus monkeys (Macaca

407 mulatta). J Comp Psychol, 114(1), 47-60.

408 Pascalis, O., \& Bachevalier, J. (1998). Face recognition in primates: a cross-species study. Behav

$409 \quad$ Process, 43(1), 87-96.

410 Platek, S. M., \& Kemp, S. M. (2009). Is family special to the brain? An event-related fMRI study

411 of familiar, familial, and self-face recognition. Neuropsychologia, 47(3), 849-858.

412 Pokorny, J. J., \& de Waal, F. B. (2009). Face recognition in capuchin monkeys (Cebus apella). J Comp Psychol, 123(2), 151-160.

414 Racca, A., Amadei, E., Ligout, S., Guo, K., Meints, K., \& Mills, D. (2010). Discrimination of human and dog faces and inversion responses in domestic dogs (Canis familiaris). Anim

416 Cogn, 13(3), 525-533.

417 Rossion, B., Schiltz, C., \& Crommelinck, M. (2003). The functionally defined right occipital and fusiform "face areas" discriminate novel from visually familiar faces. Neuroimage, 19(3), 
877-883.

420 Shepherd, S. V., Steckenfinger, S. A., Hasson, U., \& Ghazanfar, A. A. (2010). Human-monkey

422 $20(7), 649-656$.

423

Steckenfinger, S. A., \& Ghazanfar, A. A. (2009). Monkey visual behavior falls into the uncanny

424 valley. Proc Natl Acad Sci US A, 106(43), 18362-18366.

425

Tomonaga, M. (2007). Visual search for orientation of faces by a chimpanzee (Pan troglodytes):

426 face-specific upright superiority and the role of facial configural properties. Primates,

427 $48(1), 1-12$.

428

Unema, P. J. A., Pannasch, S., Joos, M., \& Velichkovsky, B. M. (2005). Time course of information processing during scene perception: The relationship between saccade amplitude and fixation duration. Vis Cogn, 12(3), 473-494. match features in familiar and unfamiliar individuals. Anim Cogn, 17(5), 1089-1105. strangers using the concept of familiarity. Animal Behaviour, 80(1), 109-115. 
436 Table 1. Participants' names, age and sex

437

\begin{tabular}{|l|c|c|c|}
\hline reference number & name & age (years) & sex \\
\hline \hline chimpanzee 1 & Hatsuka & 4 & female \\
chimpanzee 2 & Iroha & 4 & female \\
chimpanzee 3 & Loi & 17 & male \\
chimpanzee 4 & Misaki & 12 & female \\
chimpanzee 5 & Mizuki & 15 & female \\
chimpanzee 6 & Natsuki & 7 & female \\
chimpanzee 7 & Tsubaki & 16 & female \\
chimpanzee 8 & Zamba & 17 & male \\
\hline
\end{tabular}




\section{Figure legends}

440

441 Figure 1. Visual preferences of chimpanzees for different types of faces. $(a)$ An example of

442 three different types of stimuli: familiar face (i), intermediate face (ii) and novel face (iii). (b)

443 The mean-proportional fixation duration for each of the face types. (c) The mean-proportional

444 fixation count for each of the face types. (d) The mean-proportional saccade length for each of

445 the face types. Boxplots describe the responses to each type of facial stimuli. ${ }^{*} p<0.05, * * p<$

4460.01 (Friedman test).

447

448 Figure 2. Visual preferences of chimpanzees for different face types. (a) The mean proportional

449 fixation duration for each of the face types: a 100\% novel face and a 50\%-50\% morphed face

450 with different novel faces. (b) The mean proportional fixation count for each of the face types.

451 (c) The mean proportional saccade length for each of the face types. Boxplots describe the

452 responses to each type of face stimuli. n.s.: no significant difference (Wilcoxon signed-rank test). 453 
(a) (i)

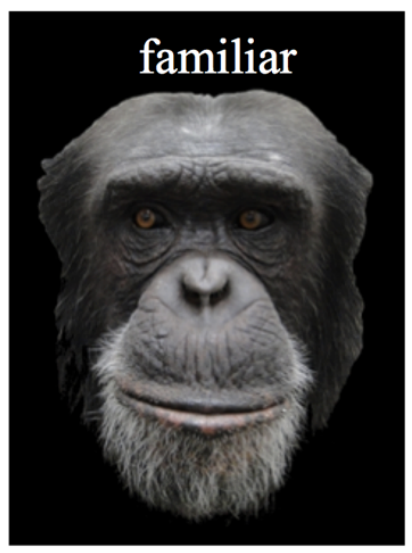

(ii)

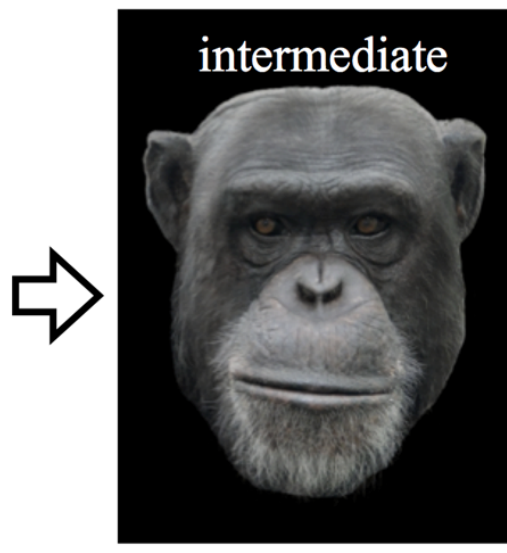

(iii)

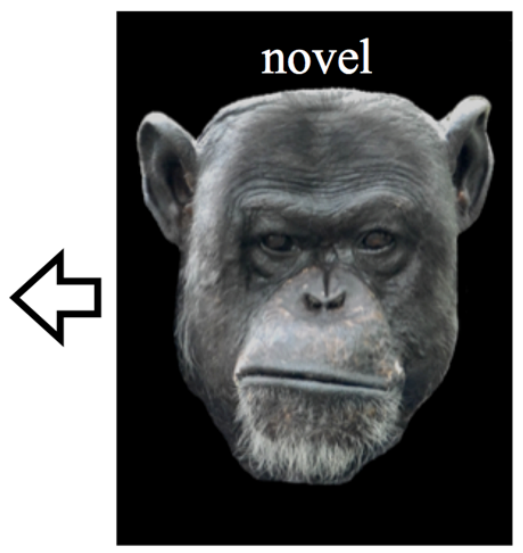

(b)

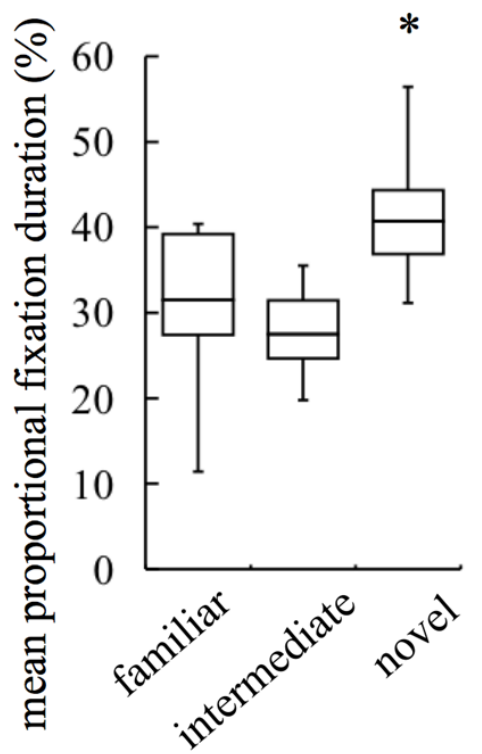

(c)

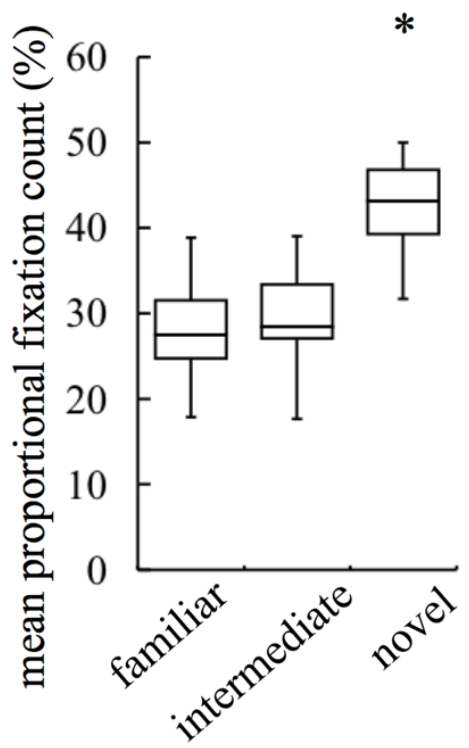

(d)

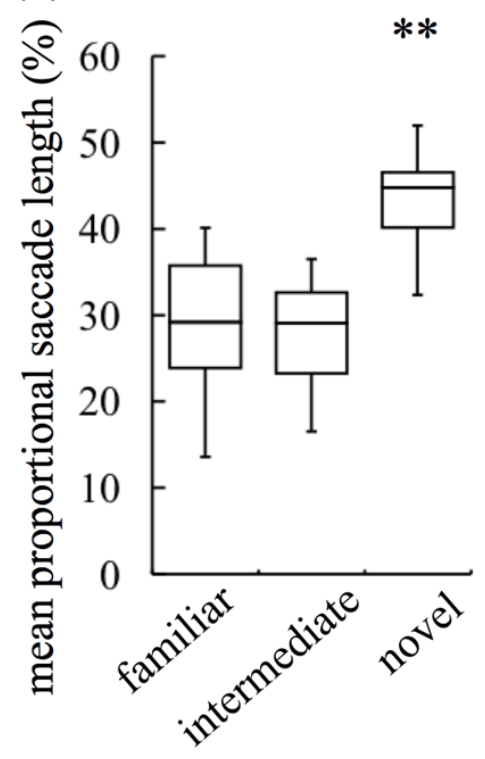


(a)

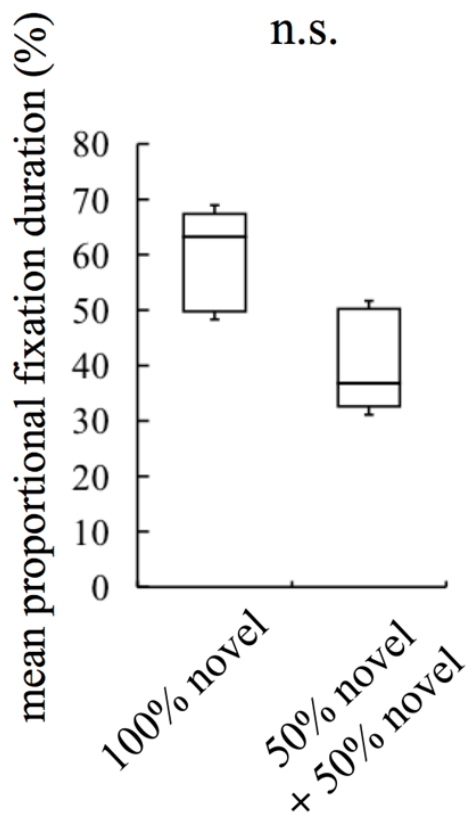

457

458

459 (b)

(c)
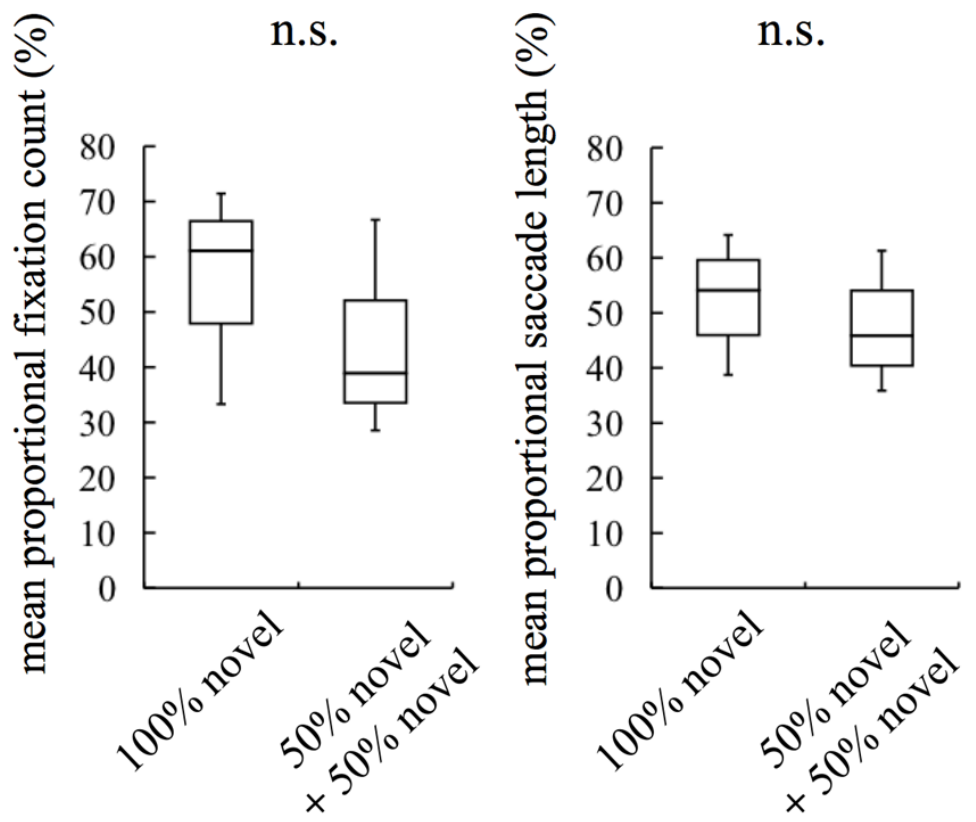

Figure 2 


\section{Table $\mathbf{1}$ (on next page)}

Participants' names, age and sex 
1 Table 1. Participants' names, age and sex

2

\begin{tabular}{|l|c|c|c|}
\hline reference number & name & age (years) & sex \\
\hline \hline chimpanzee 1 & Hatsuka & 4 & female \\
chimpanzee 2 & Iroha & 4 & female \\
chimpanzee 3 & Loi & 17 & male \\
chimpanzee 4 & Misaki & 12 & female \\
chimpanzee 5 & Mizuki & 15 & female \\
chimpanzee 6 & Natsuki & 7 & female \\
chimpanzee 7 & Tsubaki & 16 & female \\
chimpanzee 8 & Zamba & 17 & male \\
\hline
\end{tabular}

3 
Figure 1 (on next page)

Visual preferences of chimpanzees for different types of faces.

(a) An example of three different types of stimuli: familiar face (i), intermediate face (ii) and novel face (iii). (b) The mean-proportional fixation duration for each of the face types. (c) The mean-proportional fixation count for each of the face types. $(d)$ The mean-proportional saccade length for each of the face types. Boxplots describe the responses to each type of facial stimuli. $* p<0.05, * * p<0.01$ (Friedman test). 
PeerJ

\section{(a) (i)}

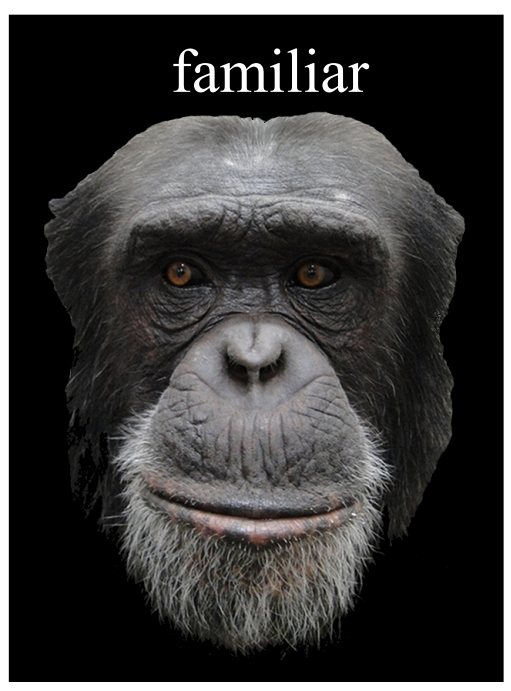

(ii)

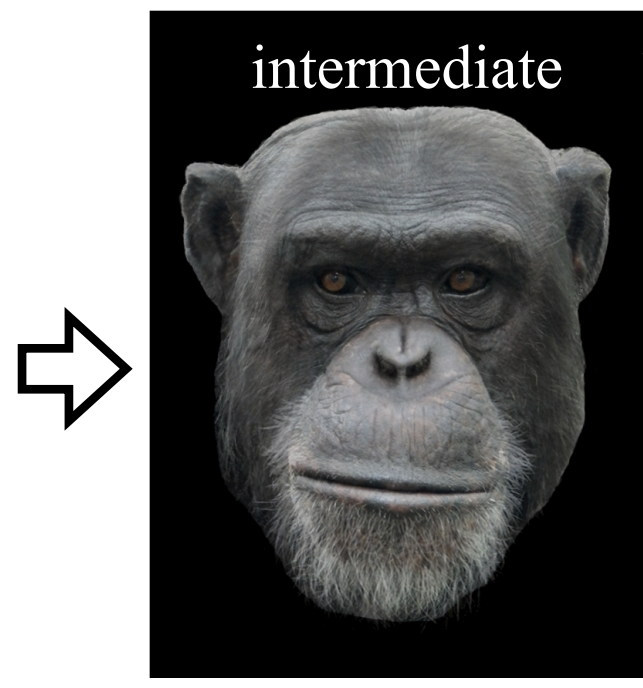

Manuscript to be reviewed

(iii)

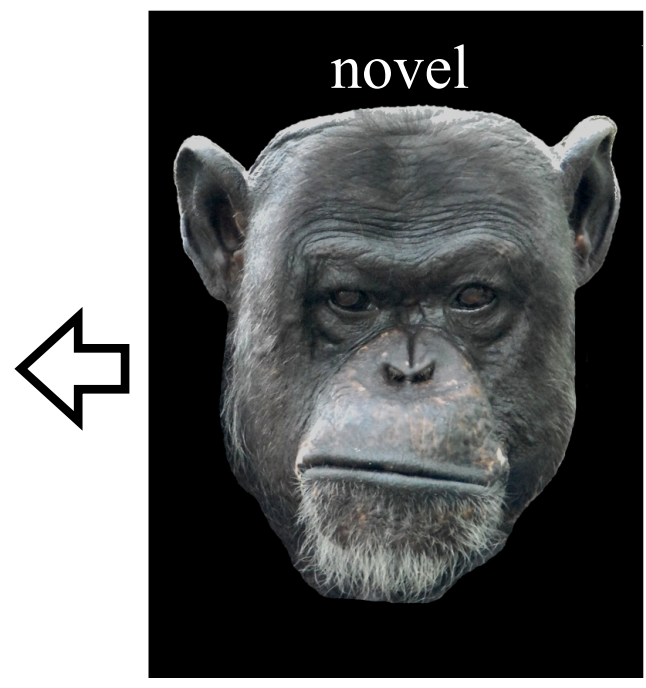

(b)

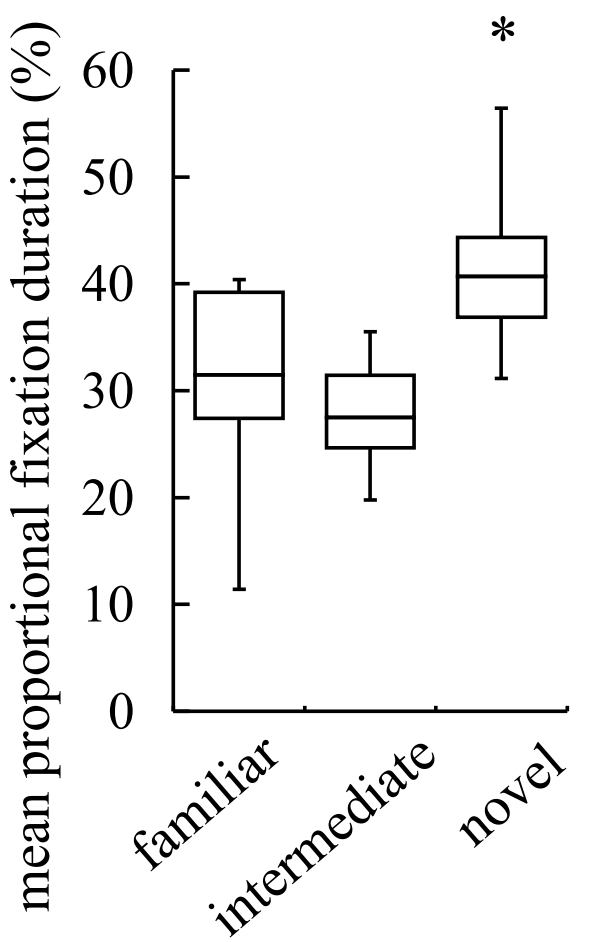

(c)

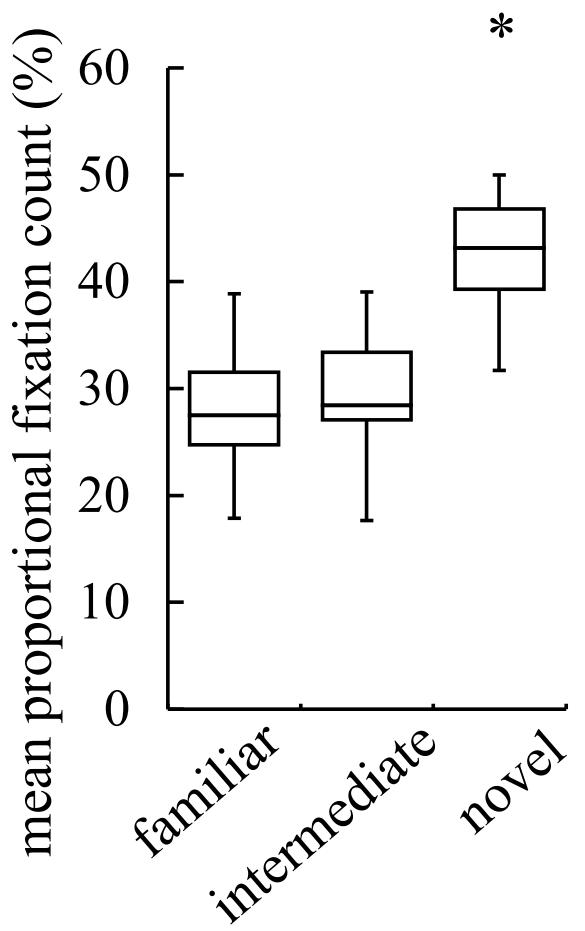

(d)

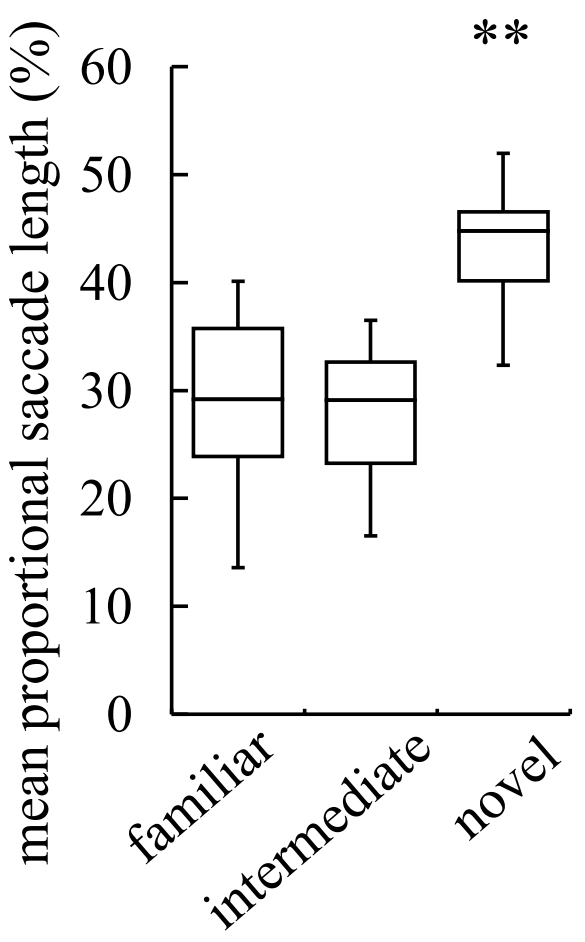


Figure 2 (on next page)

Visual preferences of chimpanzees for different face types.

(a) The mean proportional fixation duration for each of the face types: a $100 \%$ novel face and a 50\%-50\% morphed face with different novel faces. (b) The mean proportional fixation count for each of the face types. (c) The mean proportional saccade length for each of the face types. Boxplots describe the responses to each type of face stimuli. n.s.: no significant difference (Wilcoxon signed-rank test). 
(a)

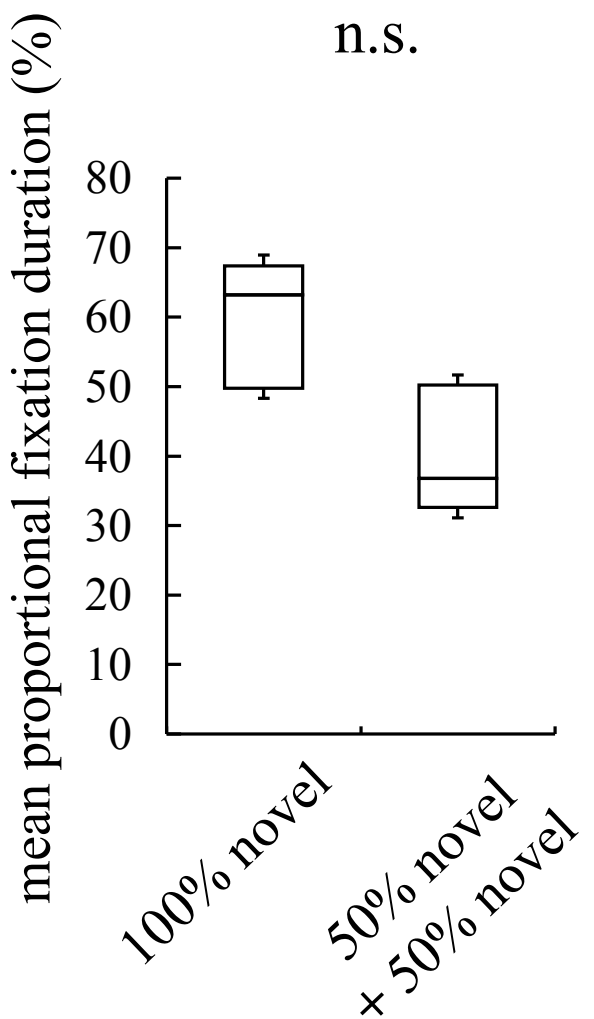

(b)

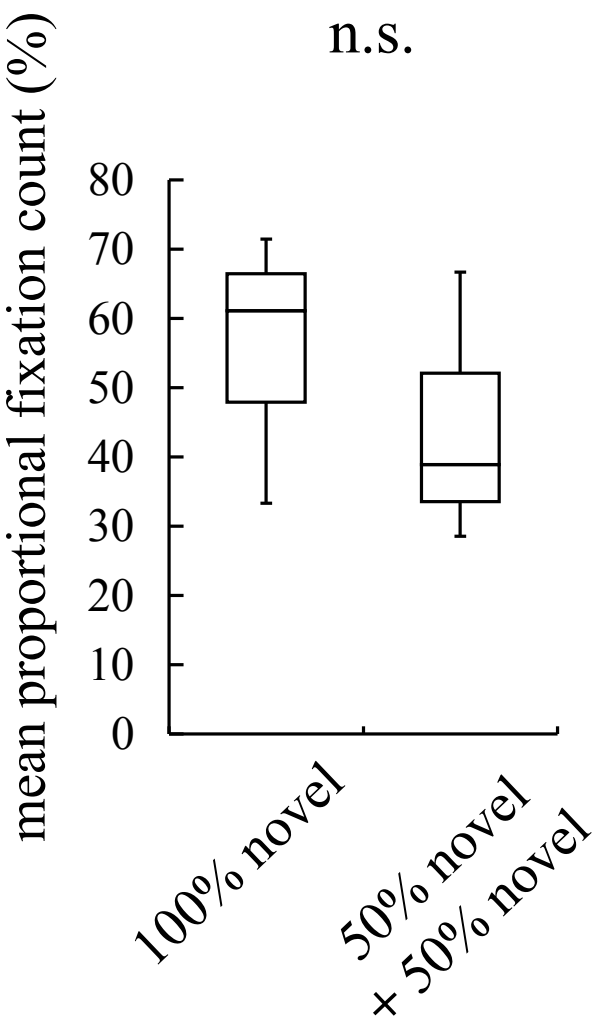

(c)

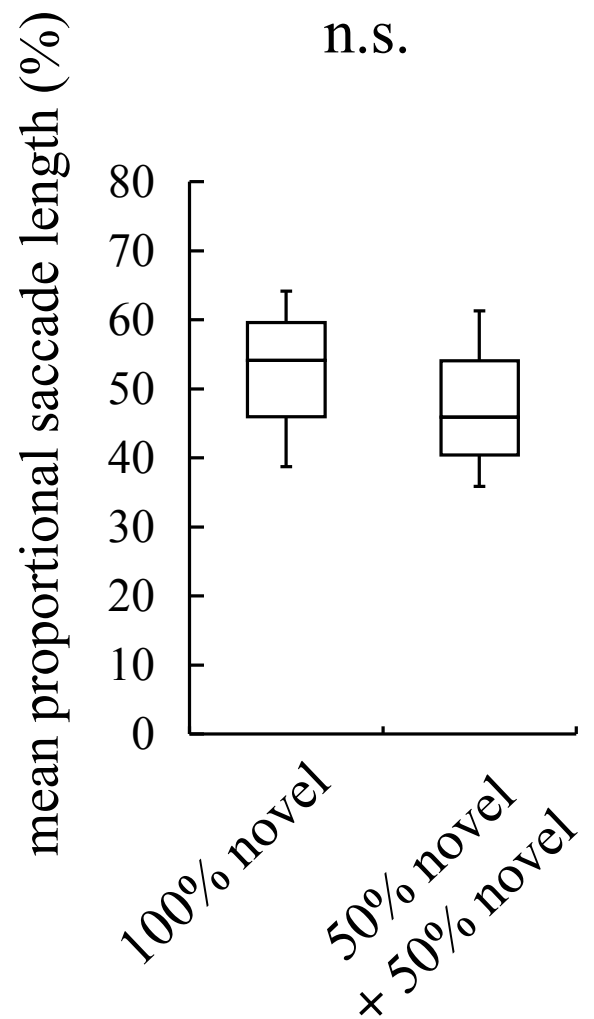

